IV. Christiansen distingue 4 phases:

0 à 1 heure - très mauvais

1 à 3 heures - mauvais

3 à 6 heures - qualité moyenne

plus de 6 heures - bon

Ainsi que le calcul statistique l'a démontré, ce classement correspond exactement à celui de BARTHEL-ORLA JENSEN pour l'épreuve au bleu de méthylène.

\title{
LES ENZYMES DU LAIT ET LEUR UTILISATION POUR APPRÉCIER LE CHAUFFAGE DU LAIT
}

\author{
par C. LIND. \\ Bactériologiste de laiterie, à Copenhague (Danemark)
}

Dans le contrôle pratique du lait, on a souvent besoin de déterminer si le lait a été chauffé ou non. C'est souvent là une chose difficile, parce que l'influence de la chaleur sur le lait varie avec la température et, d'autre part, le lait à examiner peut être un mélange de lait chauffé et de lait cru. Les questions auxquelles on doit répondre sont les suivantes :

$1^{\circ}$ Le lait a-t-il été chauffé à moins de $80^{\circ} \mathrm{C}$., c'est-à-dire pasteurisé à " haute température " comme l'exige la loi danoise, quand il est vendu sous le qualificatif "pasteurisé "?

Le lait a-t-il été chauffé momentanément à des températures inférieures à $80^{\circ}$ ?

$3^{0}$ Le lait a-t-il été chauffé pendant un temps défini à $62-64^{\circ}$, c'està-dire a-t-il subi ce qu'on appelle la "pasteurisation basse "?

$4^{\circ}$ Le lait est-il un mélange de lait chauffé et de lait cru?

$5^{\circ}$ Le lait est-il complètement cru, c'est-à-dire n'a-t-il subi aucun chauffage, quel qu'il soit?

La détermination du chauffage à $80^{\circ}$ ou au-dessus n'est pas difficile, elle s'effectue avec la méthode de STORCH (paraphénylenèdiamine et eaux oxygénée).

Mais il n'en est plus de même avec la pasteurisation basse, qui est très répandue sauf au Danemark. Un lait ainsi pasteurisé est, comme on le sait, chauffé une demi-heure à $62-64^{\circ}$. Il a partiellement conservé les qualités chimiques et biologiques du lait cru, ce que les médecins et surtout les pédiâtres considèrent comme très important pour l'élevage de l'enfance. Avec la méthode de STоRCH, il est impossible de contrôler le chauffage du lait qui a subi la pasteurisation basse, ainsi qu'un chauff age momentané à des températures inférieures à $78^{\circ}$, ou des mélanges de lait cru avec du lait ehauffé. Avee la méthode de STorcH, un lait chauffé momentanément à des températures au-dessous de $78^{\circ}$ et un lait provenant du mélange de lait pasteurisé avec au moins $10 \%$ 
de lait cru, donnent la réaction d'un lait $\mathrm{cru}$. Ils ont cependant les earactères d'un lait pasteurisé à haute température.

Les méthodes servant à déterminer si un lait a été chauffé ou non se rangent dans deux groupes :

a) Les méthodes dérivant de réactions diastasiques déclanchées par les ferments solubles du lait. Elles se basent sur ce fait que les enzymes sont affaiblies ou détruites par le chauffage. Ces méthodes sont sous la dépendance de la catalase, de l'aldéhyde-réductase (enzyme de Schardinger), de la réductase et de la peroxydase (enzyme de STORCH).

b) Une méthode chimique qui est basée sur la coagulation de l'albumine par le chauffage du lait à des températures élevées.

Les deux premières des enzymes citées ci-dessus se trouvent normalement dans le lait. Or, comme elles sont formées par beaucoup de bactéries, conséquemment les réactions auxquelles elles servent de bases ne peuvent aider à déceler le chauffage du lait que lorsque les bactéries ne sont pas très nombreuses.

En présence de lait très pollué, renfermant une grande quantité de ces bactéries, ces méthodes sont inexactes et j'ajouterai même, cela va sans dire, tout à fait inutiles; elles ne peuvent avoir quelque valeur que dans le cas des bons laits.

Or, ce que souhaite l'approvisionnement en lait, c'est une matière première qui soit pauvre en germes. Lorsque le lait est défectueux, qu'il ait été ou non chauffé au préalable, il doit être euit avant la consommation, à moins qu'il n'y soit pas livré. Si la plus grande partie du lait n'a pas été chauffée, ou si tout le lait l'a été insuffisamment, soit pendant peu de temps et à une température assez basse, il ne sera pas possible de le différencier d'un lait cru; mais dans ce cas, si le lait a conservé le caractère d'un lait cru, il sera, par ailleurs, très pollué riche en microbes et nous avons la ressource d'utiliser des réactions qui sont basées sur la présence de la réductase.

En effet, l'épreuve de la réductase, avec le bleu de méthylène, nous dira si le lait est riche ou pauvre en bactéries, la quantité de réductase présente étant sous la dépendance des microbes. En conséquence, cette épreuve doit toujours être utilisée avec les autres.

La peroxydase n'est pas d'origine microbienne ; aussi la signification de la réaction qu'elle détermine est-elle tout autre. Nous en dirons autant de l'épreuve qui est basée sur la coagulation de l'albumine, et qui ne saurait être influencée par les bactéries.

Dans ces deux conditions ces deux méthodes, l'une biologique et l'autre chimique, nous serviront aussi bien pour un mauvais lait que pour un bon lait.

Dans le Laboratoire bactériologique de la Société "Trifolium " de Copenhague; qui contribue à l'approvisionnement de cette ville, 
j'ai étudié comparativement les méthodes ci-dessus, leur valeur et voici quels sont les résultats de mes recherches:

L'épreuve de la catalase est basée sur la décomposition de l'eau oxygénée. L'oxygène qui se dégage est mesuré et sa quantité est considérée comme une mesure de la catalase du lait. Un assez grand nombre d'appareils ont été recommandés pour cette épreuve, mais les résultats qu'ils donnent ne sont pas toujours comparables. J'ai choisi l'appareil de HoyBerg, très simple et particulièrement répandu en Danemark.

Si l'on désire une exactitude plus grande, on s'adressera à l'appareil de Loвeck.

L'appareil de HoYBerg se compose d'une éprouvette avec un col d'une capacité de $12 \mathrm{~cm}^{2} 5$. On verse $2 \mathrm{~cm}^{3} 5$ d'eau oxygénée dans cette éprouvette et on la remplit de lait $\left(10 \mathrm{~cm}^{3}\right)$, puis on la renverse sur un verre rempli d'eau. On laisse pendant deux heures à une température de $20^{\circ}$ environ. Au bout de 2 heures, la plus grande partie du volume d'oxygène à dégager a été libéré et a chassé un égal volume de lait. Le volume est mesuré sur l'éprouvette qui est graduée. Le volume de l'oxygène dégagé dépend au plus haut point de la concentration de l'eau oxygénée, ainsi qu'un peut le constater dans le tableau I. Je dirai que, pour mes recherches en général, c'est la solution d'eau oxygénée à $3 \%$ que j'utilise.

\section{TABLEAU I}

Infuence de la concentration de l'eau oxygénée sur la valeur de la catalase.

\% đe l'eau oxygénée

Lait cru

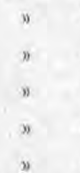

Lait pasteurisé
$3 \% \quad 1,5 \%$

$6,0 \quad 4,0$

$7,7 \quad 4,7$

$3,4 \quad 2,6$

$4,5 \quad 3,0$

$3,0 \quad 2,7$

$2,3 \quad 1,7$

$0,3 \quad 0,2$

$0,0 \quad 0,0$

1.100 échantillons de lait cru ont été examinés, quelques-uns ont été achetés en ville, mais la plupart ont été pris sur des laits frais venant de fermes, à l'arrivée à Copenhague.

Sur ces $1.100,3$ seulement nous ont donné moins de $1,5 \mathrm{~cm}^{3}$ d'oxygène ; et dans ces 3 cas l'eau oxygénée était affaiblie, aussi peut-on fixer 1,5 comme la limite inférieure de l'indice catalasimétrique dans le lait cru et si l'on-désire une limite ferme, on pourrait prendre 1,3 . Si l'on trouve des laits vendus sous le nom de laits crus ayant des valeurs inférieures à 1,3 , il faut les examiner autrement. T'ai trouvé dans cette eatégorie des laits avec des valeurs de 0,5 ; mais j'ai pu, aveo la méthode de SтоRсн, les démasquer comme des laits chauffés. 
Des laits crus contenant peu de catalase renferment toujours peu de bactéries, mais l'inverse n'est pas vrai. Des laits purs se conservant bien sans que l'on ait pu noter chez eux une augmentation considérable des bactéries pendant 24 heures à une température inférieure à $10^{\circ}$, n'ont pas vu leur catalase augmenter nettement. Si la catalase du lait augmente rapidement, e'est qu'il y a parallèlement un développement microbien vigoureux. Ce que l'on peut dire, c'est que: une grande quantité de catalase dans un lait pur, pauvre en germes, provient du lait propre et n'est pas formée par des bactéries.

$\mathrm{Si}$ le lait est chauffé pendant un moment et qu'il soit vite refroidi, la catalase diminuera d'autant plus que l'on aura chauffé à une plus haute température, ainsi qu'il résulte du tableau II.

\section{TABLEAU II}

Influence du chauffage sur l'indice catalasimétrique.

\begin{tabular}{|c|c|c|c|c|c|c|c|c|c|c|c|}
\hline No des laits & I. & II & III & IV & $\mathbf{v}$ & VI & VII & VIII & IX & $\mathrm{x}$ & XI \\
\hline Cru. & 4,0 & 4,6 & 3,3 & 4,0 & 3,4 & 3,7 & 3,6 & 3,5 & 4,3 & 8,0 & 9,0 \\
\hline Chauffé à $60^{\circ}$ & 3,0 & 4,0 & & & 2,7 & 2,9 & & & & & \\
\hline $62^{\circ}$ & & & 1,8 & 2,5 & & & & 1,5 & & & \\
\hline $65^{\circ}$ & 0,5 & 2,8 & 0,5 & & 0,7 & 2,0 & & & 2,5 & & \\
\hline $70^{\circ}$ & 0,3 & 1,5 & 0,2 & 0,9 & 0,0 & 0,2 & 0,5 & 0,7 & 0,2 & 0,7 & 2,7 \\
\hline $75^{\circ}$ & 0,0 & 0,0 & 0,0 & 0,4 & 0,0 & 0,0 & 0,0 & 0,2 & & & \\
\hline$n \quad 80^{\circ}$ & 0,0 & 0,0 & 0,0 & 0,0 & 0,0 & 0,0 & 0,0 & 0,0 & 0,0 & & \\
\hline
\end{tabular}

Des 11 échantillons du tableau II, les $\mathrm{n}^{\mathrm{os}} \mathrm{II}$, IV etIX étaient des laits d'une qualité inférieure, Les $n^{08} \mathrm{X}$ et XI étaient des laits très mauvais.

Le tableau II montre, en outre, qu'un lait chauffé à $80^{\circ}$ voit sa catalase détruite et s'il est bien conservé, après chauffage, les bactéries ne se développant pas, eette eatalase ne réapparsîtra pas. Au contraire, si on donne aux bactéries l'occasion de se développer à nouveau, la catalase augmente et on peut dire que la valeur de l'indice catalasimétrique de laits pasteurisés au-dessus de $80^{\circ}$ est une mesure peu exacte du développement ultérieur des bactéries, puisque les recherches d'OrLA JENSEN nous ont montré que les diverses espèces bactériennes secrètent des quantités de calatase différentes.

Si le lait est chauffé pendant un temps plus ou moins long à une température plus basse, la catalase diminue en proportion de la durée du chauffage.

Dans quelques expériences que j'ai faites, où j'ai chauffé du lait pendant 20-30 minutes à $60^{\circ}-64^{\circ}$, l'indice catalasimétrique de bons laits ainsi traités était descendu au-dessous de 1,5, limite inférieure du lait cru, mais dans le cas de laits très impurs, ils ne descendaient pas au-dessous de cette limite, ainsi qu'il résulte de l'examen du tableau III. 


\section{TABLEAU III}

Influence de la durée du chauffage sur l'indice catalasimétrique

\begin{tabular}{|c|c|c|c|c|c|c|c|c|c|c|c|}
\hline No des laits & I & II & III & IV & v & VI & VII & VIII & IX & $\mathrm{x}$ & XI \\
\hline Température & $64^{\circ}$ & $\begin{array}{l}60- \\
62^{\circ}\end{array}$ & $\begin{array}{l}60- \\
62^{\circ}\end{array}$ & $62^{\circ}$ & $\begin{array}{l}62- \\
64^{\circ}\end{array}$ & $\begin{array}{l}63- \\
65^{\circ}\end{array}$ & $\begin{array}{r}62- \\
64^{\circ}\end{array}$ & $62^{\circ}$ & $\begin{array}{l}60- \\
63^{\circ}\end{array}$ & $\begin{array}{l}60- \\
63^{\circ}\end{array}$ & $\begin{array}{l}62- \\
63^{\circ}\end{array}$ \\
\hline Lait cru. . . & 4,0 & & 3,5 & 3,9 & 3,5 & & 4,0 & 5,2 & 3,0 & & \\
\hline $\begin{array}{l}\text { Chauffé mo- } \\
\text { mentanément. }\end{array}$ & 2,4 & 3,2 & & 3,0 & 1,5 & & & & & & \\
\hline Chauffé $5 \mathrm{~min}$. & 1,4 & & & & & & & & & & \\
\hline " $12 \mathrm{~min}$. & & 1,7 & 1,3 & 1,3 & 1,1 & & & & & & \\
\hline $15 \mathrm{~min}$. & 1,0 & & & & & & & & & & \\
\hline $20 \mathrm{~min}$. & & 1,0 & 1,0 & & 0,8 & & & & & & \\
\hline $30 \mathrm{~min}$, & 0,8 & 1,0 & 0,8 & 0,9 & 0,8 & 0,7 & 0,8 & 2,0 & 1,0 & 1,5 & 1,0 \\
\hline
\end{tabular}

Les échantillons du tableau III, les $n^{\circ}$ VIII et X étaient extrêmement défectueux, les autres étaient bons ou médiocres.

$\mathrm{Si}$ un lait chauffé à $60-64^{\circ}$ pendant 20-30 minutes est conservé pendant 24 heures à des températures au-dessous de $10^{\circ}$, l'indice catalasimétrique n'augmente pas ou, du moins, s'il le fait, ce n'est que d'une façon très insignifiante. Ce fait est dû à ce que les bactéries ne se développent pas.

L'épreuve de Schardinger est basée, comme on le sait, sur la réduction du bleu de méthylène en présence de formol. L'enzyme qui interviendrait a été appelé aldéhyde-réductase. J'ai eu recours à cette épreuve en utilisant la solution ordinaire de bleu de méthylène, telle qu'elle est employée dans la méthode de la réductase, avec une addition de $2,5 \%$ de formol. A $10 \mathrm{~cm}^{3}$ de lait, on ajoute $0,5 \mathrm{~cm}^{3}$ dela solution bleu de méthylène formolée et on laisse au B-M à $48-50^{\circ}$.

ScHARDINGER estime qu'un lait cru est décoloré avant 10 minutes et un lait cuit seulement après 2 heures. Cette opinion a été corroborée par d'autres savants et mes recherches ne sauraient que les confirmer. Cependant ScherN aurait trouvé des vaches qui, au commencement de la lactation, produisaient un lait qui ne décolore la solution de Schardinger qu'après 10 minutes? ORLA JENSEN de son côté a trouvé 2 échantillons qui se décolorent après 10 minutes, mais il ne dit pas à quelle période de la lactation ils correspondaient. BARTHEL a trouvé également quelques valeurs au-dessus de 10 minutes, mais la plupart sont au-dessous.

De mon côté, j'ai examiné plus de 200 échantillons de laits erus qui se décolorent avant 10 minutes. L'aldéhyde-réductase se trouve dans le lait propre, mais elle est également formée par les bactéries, ce qui est démontré de la façon suivante : 
a) Avec l'âge du lait, la durée de la décoloration est diminuée ;

b) Le lait pasteurisé qui, aussitôt après la pasteurisation ne décolore qu'au. bout de quelques heures, décolore en quelques minutes quand les bactéries ont eu l'occasion de pousser vigoureusement.

Dans le tableau IV, on va pouvoir apprécier l'influence du chauffage sur le temps de décoloration dans la réaction de SchardingER, lequel s'accroît parallèlement avec le chauffage.

\section{TABLEAU IV}

L'influence du chauffage sur la valeur du Schardinger

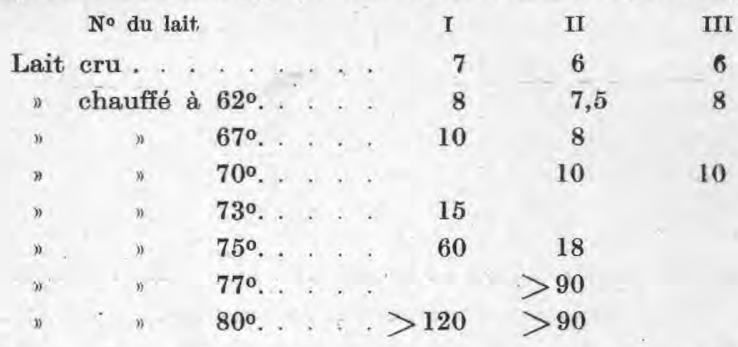

Alors que dans le tableau IV, il s'agissait d'un chauffage rapide, une minute ou deux, dans le tableau $V$ il s'agit d'un chauffage à 60-64 pendant des temps variables allant jusqu'à 30 minutes.

\section{TABLEAU V}

L'influence de la durée du chauffage sur la valeur du Schardinger

\begin{tabular}{|c|c|c|c|c|c|c|c|c|c|c|c|}
\hline$N^{\circ}$ du lait & I & II & III & IV & V & VI & VII & VIII & IX & $\mathrm{x}$ & XI \\
\hline Température & $62^{\circ}$ & $\begin{array}{l}62- \\
63^{\circ}\end{array}$ & $\begin{array}{l}62- \\
64^{\circ}\end{array}$ & $\begin{array}{l}62- \\
64^{\circ}\end{array}$ & $\begin{array}{l}60- \\
64^{\circ}\end{array}$ & $\begin{array}{l}62- \\
64^{\circ}\end{array}$ & $\begin{array}{l}62- \\
64^{\circ}\end{array}$ & $62^{\circ}$ & $\begin{array}{l}60- \\
63^{\circ}\end{array}$ & $\begin{array}{l}60- \\
63^{\circ}\end{array}$ & $\begin{array}{l}60- \\
64^{\circ}\end{array}$ \\
\hline Lait cru . . . . & 6 & 7 & 6 & 8 & 9 . & 5 & 6 & 2,5 & 9 & 2 & 6 \\
\hline Durée : $0 \mathrm{~min} .$. & 8 & 8 & 7,5 & 7 & & & & & & & \\
\hline 10 & 12 & 9 & 8,5 & 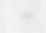 & & & & & & & \\
\hline 20 & & 11 & 9 & & & & & & 4 & & \\
\hline 30 & 17 & 13 & 11,5 & 11 & 10 & 8 & 9,5 & 6,5 & 8 & 8 & 9 \\
\hline Secoué . . . & 23 & 15 & 14 & 13 & 16 & 13 & 18 & 9,5 & 13 & 17 & 14 \\
\hline
\end{tabular}

Dans les deux tableaux qui précèdent, quelques valeurs pour les laits chauffés sont trop basses. Il faut en trouver l'explication dans ce que le gaz absorbé par le lait pendant le chauffage tend à retarder considérablement la décoloration. Ainsi un lait cru après passage sur un réfrigérant qui a permis de le mettre en contact sur une large surface avec l'air a augmențé de 2 minutes le temps de la décoloration. Si un lait chauffé est agité avec de l'air, ou s'il passe également sur un réfrigérant, il absorbe de l'air, ce qui augmente beaucoup le temps de décoloration. Dans l'épreuve de SchardingER, ce n'est qu'après cette 
absorption d'air que les valeurs dans les réactions avec les laits chauffés peuvent être comparables avec celles que donnent les laits crus, lesquels contiennent toujours de l'air.

Dans le tableau V, sous la désignation "secoué " sont indiquées les valeurs données par des laits pasteurisés à basse température après qu'ils ont été agités avec de l'air. Tous ces échantillons, à l'exception du VIII, ont des valeurs au-dessus de 10, c'est-à-dire au-dessus de la valeur des laits crus. Notons en passant que le lait VIII est un lait très mauvais. BARTHEL avait déjà vu qu'un lait pasteurisé à basse température se décolorait avec le réactif de SCHARDINGER plus lentement lorsqu'il est refroidi en passant sur un réfrigérant que s'il l'est dans le récipient avec de la glace et il estime que e'est l'air absorbé qui est la cause de ce phénomène. La conclusion de BARTHEL est que l'aldéhyderéductase est affaiblie seulement, mais non détruite par une telle pasteurisation.

Il faut distinguer ici entre les laits récemment traits et les laits qu'on a l'occasion d'examiner dans le contrôle ordinaire, qui sont plus âgés et altérés et dont toutes les valeurs sont au-dessous de 10 .

L'épreuve de la réductase s'exécute ainsi, d'après BARTHEL et ORLAJENSEN : $40 \mathrm{~cm}^{3}$ de lait sont mélangés avec $1 \mathrm{~cm}^{3}$ d'une solution aqueuse de tablettes de bleu de méthylène de Blauenfeld et Tvene, laissés au repos au B-M à $38-40^{\circ}$ et observés par intervalles. Le nombre d'heures qui s'écoulent jusqu'à ce que la décoloration soit complète est nommè la valeur de la réductase. Plus il y a de bactéries dans le lait, plus petite est cette valeur et inversement. La richesse bactérienne d'un lait n'est pas exactement en raison inverse du temps de la décoloration, car, ainsi que l'a montré ORLA-JENSEN, différentes bactéries ont des pouvoirs divers de réduction. Mais la méthode, qui a été très bien examinée dans la pratique, est aujourd'hui tellement appréciée qu'on peut dire en toute confiance que l'épreuve de la réductase, en dépit des eritiques théoriques qu'on peut lui adresser, est extrêmement utile pour classer les laits comme bons, médiocres et impurs (BARTHEL, LIND). Si la richesse bactérienne d'un lait ne dépasse pas 500.000 bactéries par $\mathrm{cm}^{3} \mathrm{le}$ temps de réduction du lait sera au moins de 5 h. à 5 h. 1/2. S'il est de 2 heures; le lait est considéré comme impur. Un lait qui décolore entre 2 et 5 heures est médiocre. Si l'on veut savoir plus exactement le nombre de bactéries du lait, on sait qu'il faut des méthodes plus coûteuses, recourir à des ensemencements sur des plaques de gélatine ou d'agar, ce qui, dans la plupart des cas, ne saurait être fait.

L'épreuve de Storch se réalise de la façon suivante : Dans une éprouvette, on verse 5 à $10 \mathrm{~cm}^{3}$ de lait, puis I goutte de solution aqueuse d'eau oxygénée contenant $0,2 \%$ d'eau oxygénée et ensuite II gouttes d'une solution aqueuse de paraphénylène-diamine à $1 \%$ 
fraîchement préparée. Après mélange, le lait cru prend très vite une couleur bleu-indigo. Un lait qui a subi le chauffage à des températures au-dessus de $80^{\circ}$ conserve sa couleur blanche. Si le lait, au bout d'une demi-heure, prend une tonalité plus bleuâtre, e'est qu'il a été chauffé à une température un peu inférieure à $80^{\circ}$ ou qu'il résulte d'un mélange de lait pasteurisé avec une quantité de lait cru ne dépassant pas 5-10\%. Si un lait a été chauffé momentanément à des températures au-dessous de $78^{\circ}$ ou si le lait pasteurisé est additionné d'une- quantité de lait eru dépassant $10 \%$, la réaction qu'on obtient est celle du lait cru.

Avec l'épreuve de SтоRch, il est done impossible de dire si un lait a été chauffé à une tempérautre plus basse que $78^{\circ}$ ou s'il n'a pas subi le moindre chauffage.

L'épreuve de l'albumine. - Il y a plusieurs moyens de l'appliquer. J'accorde ma préférence à celle de SoxнLEт, qui est basée sur la coagulation de la caséine par acidulation sans chauffage. Dans le lait cru, l'albumine reste en solution et on la précipitera quand on chauffera le sérum préalablement obtenu. Si le lait est pasteurisé à haute température, ou préalablement bouilli, l'albumine aura été précipitée presque totalement et conséquemment lorsqu'on chauffera le filtrat obtenu après précipitation de la caséine par un acide, on n'obtiendra qu'une précipitation faible ou même seulement qu'un léger trouble.

L'épreuve peut être exécutée comme suit : $10 \mathrm{~cm}^{3}$ de lait sont dilués avec $40 \mathrm{~cm}^{3}$ d'eau, puis on précipite à froid à l'aide de $2 \mathrm{~cm}^{3}$ d'une solution à $3 \%$ d'acide acétique. Le filtrat est chauffé quelques instants à $85-90^{\circ}$. Du lait cru ou du lait pasteurisé à basse température donnera un précipité considérable, tandis qu'avec un lait pasteurisé à haute température, il n'y aura qu'un trouble; le filtrat se troublera faiblement.

$\mathrm{Si}$ on ehauffe le filtrat plus longtemps à $85-90^{\circ}$ à une température plus élevée, on aura un petit précipité, car la pasteurisation ordinaire ne coagule pas toute l'albumine (ORLA-JENSEN).

Une détermination quantitative de l'albumine dans le sérum acétique pourrait donner des résultats sur l'importance du chauffage, mais ils seraient parfois aléatoires.

A l'aide de l'épreuve de l'albumine, on peut déceler l'addition du lait cru à du lait pasteurisé si cette addition dépasse $20.40 \%$; mais il faut reconnaître que dans ces conditions, il serait préférable de recourir à la méthode de SтоRCH.

Nous allons voir maintenant comment il faut interpréter les résultats des épreuves dont il vient d'être question, relativement à l'influence du chauffage.

Lait cru. - Avec la méthode de SтоRсн, on aura une coloration rapide et vive. L'indice catalasimétrique n'est pas inférieur à 1,5; le temps de décoloration de la réaction de ScharDinger est au maximum 
de 10 minutes; l'épreuve de la réductase nous donnera approximativement le nombre de bactéries.

Si la valeur de l'indice catalasimétrique et celle de l'épreuve de Schardinger sont en dehors des limites que donne un lait eru, on doit se demander si le lait n'a pas été chauffé, surtout si, de plus, la valeur de la réductase est petite. Très souvent on aura une réponse à cette question, en laissant le lait à une température au-dessous de $10^{\circ}$ pendant 24 heures. Après un tel repos, la valeur de la réduetase et celle de la réaction de Schardinger d'un bon lait cru, ne seront que faiblement altérées, tandis que la valeur de la catalase aura un peu augmenté.

Si le lait est moins bon, il y aura une diminution dans la valeur de la réductase, ainsi que dans celle de SCHARDINGER, et une augmentation de l'indice eatalasimétrique.

Un lait cru qui réduit rapidement le bleu de méthylène parce qu'il renferme beaucoup de bactéries, voit s'élever son indice eatalasimétrique, baisser au contraire le temps de décoloration dans la réaction de SCHARDINGER.

Lait pasteurisé au-dessus de $\mathbf{8 0}$. - Avec la réaction de STORCH, pas de coloration, ce qui suffit pour classer ce lait comme pasteurisé. Avec l'épreuve de l'albumine, il n'y a qu'un nuage dans le filtrat.

Si le lait est bon et frais, la valeur de la catalase est zéro, la valeur de la réaction de ScHARDINGER, dépasse quelques heures et la décoloration du bleu de méthylène également.

Si les bactéries se développent à nouveau dans un tel lait, des enzymes sont fabriquées et conséquemment, alors que nous voyons l'indice catalasimétrique s'élever, les temps de décoloration du bleu de méthylène et du bleu de méthylène formolé diminuent.

Mélange de lait cru avec du lait chauffé. - Du lait pasteurisé à $80^{\circ}$ additionné de 5-10\% de lait eru et un lait chauffé momentanément à $78-80^{\circ}$ donneront une coloration plus faible et ils la donneront plus lentement, avec le réactif de STоRсн. Si la proportion de lait eru est plus grande ou si le chauffage n'a pas atteint 78, l'épreuve de SтоRcH ne distingue pas un tel lait du lait cru.

L'épreuve de l'albumine n'indiquera l'addition de lait cru ou de lait pasteurisé que lorsqu'elle atteindra $40 \%$ au moins. Du lait pasteurisé avec une addition de lait cru de moins de $30 \%$ et un lait chauffé audessous de $80^{\circ}$ n'auront qu'un petit indice catalasimétrique et de grandes valeurs pour l'épreuve de la réductase et celle de ScHaRdinger. Si le chauffage dépasse $75^{\circ}$, l'indice catalasimétrique sera le plus souvent zéro, ce qui n'est pas le cas pour un lait de mélange. Les réactions d'un lait chauffé sont assez stables, celles au contraire des laits de mélange se modifient vite, puisqu'ils se conservent mal. 
Lait pasteurisé à basse température. - Avec l'épreuve de STORCH et l'épreuve de l'albumine, on ne saurait distinguer ce lait du lait cru. Si l'on a pasteurisé du bon lait et s'il est pasteurisé depuis peu de temps, son indice catalasimétrique ne dépasse pas 1,5 et le temps de décoloration de SCHARDINGER est au moins 10 , si le lait a été saturé d'air après le chauffage, par passage sur un réfrigérant. La valeur du temps de décoloration du bleu de méthylène sera toujours grande.

Si le lait n'était pas bon avant la pasteurisation, les épreuves cidessus auront de la peine à le distinguer du lait cru; et ce n'est que la relative fixité des différentes valeurs de ces épreuves après un certain temps de conservation qui le démasquerait comme du lait chauffé. En effet, une conservation au-dessous de $10^{\circ}$ pendant $24-48$ heures ne change guère ces valeurs, tandis que pour un bon lait cru, elles sont légèrement modifiées et beaucoup pour un mauvais lait cru.

Le tableau VI donne les résultats qu'on obtient avec quelques échantillons de laits traités dans des conditions différentes:

TABLEAU VI

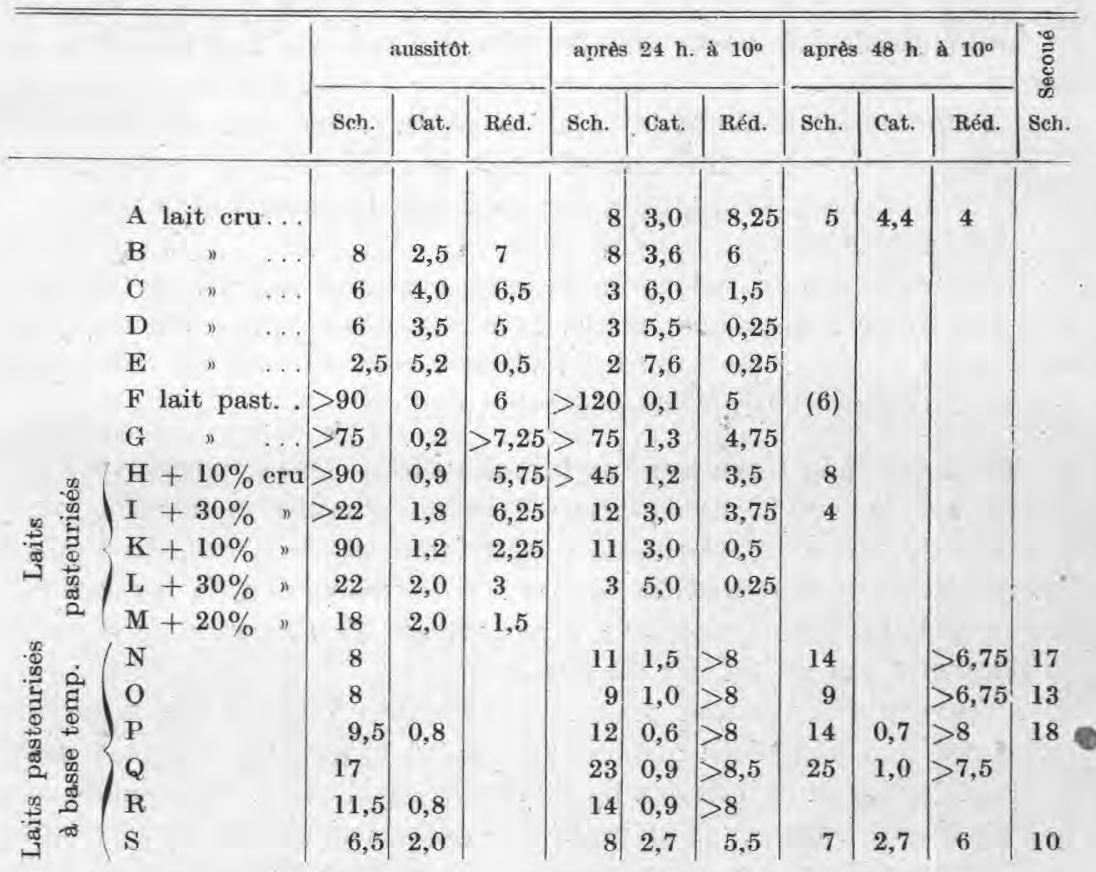

Ce tableau nous montre comment on peut apprécier à l'aide des méthodes ci-dessus examinées quel est le traitement qu'a subi le lait.

Les échantillons A et B sont des laits extrêmements bons, pauvres en germes, $\mathrm{C}$ et $\mathrm{D}$ des laits bons, $\mathrm{E}$ un lait mauvais, tous à l'état cru. 
$A$ et $B$ sont toujours bons après 24 heures. A l'est encore après 48 heures, tandis que $\mathrm{C}$ et $\mathrm{D}$ sont mauvais déjà après 24 heures. $\mathrm{E}$ est si riche en bactéries, ainsi qu'en témoigne le temps petit qu'il prend pour décolrer le bleu de méthylène, que l'indice catalasimétrique et la valeur du Schardinger ne permettent pas de nous donner des renseignements sur le traitement qu'il a subi. Les chiffres trouvés ressemblent à ceux que nous donne le lait L après 24 heures. L était un lait de mélange de lait pasteurisé et de lait cru.

Les laits pasteurisés $\mathrm{F}$ et $\mathrm{G}$ ont des chiffres élevés pour le Schardinger et bas pour l'indice catalasimétrique. La valeur de la catalase de G après 24 heures a crû, mais parallèlement, l'épreuve de la réductase nous montre que le nombre de bactéries a également augmenté. Les laits $\mathrm{H}$ à $\mathrm{M}$ sont des laits de mélange de laits pasteurisés à haute température avec des quantités variables de laits crus. Les chiffres du Schardinger sont hauts, ceux de l'indice catalasimétrique petits.

Au bout de 24 heures, ils sont tous fortement altérés.

Le lait $\mathrm{L}$ est si riche en microbes que, au bout de 24 heures, les méthodes qui ont recours aux enzymes ne peuvent plus être employées ; seule l'épreuve de l'albumine décèlerait cet échantillon comme un lait pasteurisé.

Relativement aux laits pasteurisés à basse température $\mathrm{N}$ à $\mathrm{S}$, il est étonnant de voir que les chiffres du Schardinger s'élèvent au-dessus de 10 , après qu'ils ont été secoués en présence d'air atmosphérique. D'autre part, les chiffres du Schardinger ne sont pas élevés, très audessous des chifres correspondants des laits pasteurisés à haute température. Les indices catalasimétriques sont faibles excepté celui de $\mathrm{N}$.

$\mathrm{N}$ provenait d'un lait mauvais. Il en est de même d'ailleurs de S, qui ne peut pas être distingué d'un bon lait cru ; pourtant la constance des valeurs obtenues après 48 heures nous amène à penser qu'il n'est pas cru. Il est d'importance pour le contrôle moderne du lait de savoir si le lait répond bien à la désignation sous laquelle il est mis en vente, tout au moins lorsqu'il s'agit du lait de choix, toujours coûteux.

Or, aucune des méthodes dont nous venons de parler ne nous permet de donner une certitude complète sur le chauffage et surtout sur l'importance du chauffage du lait. Il faut les combiner pour avoir des résultats plus précis (1).

\section{BIBLIOGRAPHIE}

[1] BARTHEL Chr. Langtidspasteurisering av Mjölk. Meddelande Nr. 117 fran Centralanstalten för försöksväsendet pa jordbruksomradet, 1915.

(1) Ce travail a paru autrefois on danois dans le Maanedskrift for Dyrlaeger 1917. II •était rédigé lorsque j'ai eu l'occasion de lire un rapport de.BuTTENBERGER de 1906 proposant de combiner la méthode de SchaRDINGER avec la méthode au gaĩac et la méthode de réductase d'une manière à peu près semblable à celle que j'indique pour déterminer si le lait * été cheuffé ou non et dans quelles proportions. 
[2] OrLa-Jensen. Moelkepasteuriseringsspörgsmaalet i moderne Belysning Mólkeritidende, 1916.

[3] Orta-Jensen. De l'origine des oxydases et réductases du lait de vache. Revue générale du lait, 1906.

[4] Schardinger. Ueber das Verhalten der Kuhmilch gegen Methylenblau. Zeitschrift für Untersuchung der Nahrungs- und Genussmitteln, 1902.

[5] SCHERN K., Ueberdie Schardinger Reaktion der Milch. Biochem. Zeitschrift, 1909.

[6] OrLa-Jensen. Mrelkeri-Bakteriologi.

[7] LIND C. Reduktasepröven sammenlignet med Bakterie.Tcelningsmetoden. Mcelkeritidende, 1915.

[8] BaRthel Chr. Fortsatta Undersökningar rörande Reduktasprovet. Meddelande - Nr. 141 fran Centralanstalten f. försöksväsendet pa jordbruksomradet, 1917.

[9] OrLa-JENSEn, De l'action du chauffage sur le lait de vache. Revue générale du lait, 1905.

[10[ WEBER. Die zur Unterscheidung roher und gekochter Milch dienenden Untersuchungsmetoden. Diss. Leipzig, 1902.

[11] BUttenberger. Zur Unterscheidung der pasteurisierten Milch. Zeitschrift f. Unters, der Nahrungs- u. Genussmitteln, 1906.

[12] Jensen C. O. Moelk og Moelkekontrol, 1903.

[13] Sommerfeld. Handbuch der Milehkunde, 1909.

\title{
RECHERCHES SUR L'INFLUENCE DE LA SÉCRÉTION LACTÉE SUR L'ORGANISIVIE, LE SYNDROME HYPOGLYCÉMIQUE ET LA FIËVRE VITULAIRE DE LA VACHE
}

\author{
par M. L. AUGER \\ Docteur-Vétérinaire, Chef de travaux de clinique à l'Ecole vétérinaire de Lyon
} (Suite et fin)

Le syndrome hypoglycémique obtenu expérimentalement chez la vache se traduit par des symptômes identiques à ceux de la fièvre vitulaire. - Si nos idées sont exactes et si la fièvre vitulaire est la conséquence d'un épuisement du glucose immédiatement utilisable de l'organisme, on doit pouvoir en créant artificiellement cet état chez une vache saine reproduire tous les signes de la fièvre vitulaire. L'épuisement de l'organisme en glucose immédiatement utilisable peut être décelé par l'abaissement du taux du sucre du sang, mais il se traduit aussi par des symptômes particuliers dont l'ensemble constitue ce que l'on appelle aujourd'hui le «syndrome hypoglycémique ». A la vérité ce syndrôme avait pu être obtenu autrefois par l'injection d'une substance provoquant de la glycosurie : la phloridzine dont le mode d'action est d'ailleurs encore discuté et l'ensemble des signes présentés par les animaux était connu sous le nom d'"intoxication glycoprive", mais les résultats donnés par la phloridzine étaient inconstants et e'est surtout depuis l'extraction de l'insuline par les Canadiens qu'une telle expérimentation est devenue facile. 